\title{
Prácticas sexuales de riesgo, derechos sexuales y reproductivos en una muestra de adolescentes del sureste de México
}

\section{Risky sexual practices, sexual and reproductive rights in a sample of adolescents from southeastern Mexico}

DOI: $10.46981 /$ sfjhv2n4-008

Received in: Oct 1st, 2021

Accepted in: Dec 31th, 2021

\author{
Gabriela Isabel Pérez-Aranda \\ Doctorado en Educación Humanista - Instituto Humanista de Sinaloa, México. \\ Profesora Investigadora de la Facultad de Humanidades de la \\ Universidad Autónoma de Campeche \\ Avenida Agustín Melgar, Buena Vista. C.P. 24039. \\ San Francisco de Campeche, México. \\ E-mail: gaiperez@uacam.mx \\ Sinuhé Estrada-Carmona \\ Doctorado en Ciencias Sociales - Universidad Autónoma de Yucatán, México. \\ Profesor Investigador de la Facultad de Humanidades y colaborador del \\ Laboratorio de Atención Psicológica e Investigación Clínica de la \\ Universidad Autónoma de Campeche \\ E-mail: sestrada@uacam.mx \\ Kenya Gabriela Masté-Chablé \\ Licenciada en Psicología - Facultad de Humanidades \\ Universidad Autónoma de Campeche \\ Edwin Iván Noriega-Aguilar \\ Licenciado en Psicología - Facultad de Humanidades \\ Universidad Autónoma de Campeche
}

\section{RESUMEN}

El objetivo fue analizar el conocimiento de los derechos sexuales y reproductivos y las prácticas sexuales de riesgo en una muestra de adolescentes del Estado de Campeche, en el sureste de México; para lo cual se aplicó un cuestionario ad hoc con la finalidad de recabar información sobre conductas sexuales de riesgo y conocimiento sobre derechos sexuales y reproductivos a una muestra de 490 adolescentes estudiantes de bachillerato. Se encontraron relaciones estadísticamente significativas entre el conocimiento de derechos sexuales y reproductivos con el consumo de sustancias; así como entre el riesgo de embarazos no planeados, el riesgo de contraer ITS y de recibir violencia. No se encontraron diferencias estadísticamente significativas en el análisis de comparación entre sexos.

Palabras clave: Prácticas sexuales de riesgo, derechos sexuales, derechos reproductivos, adolescentes.

\section{ABSTRACT}

The objective was to analyze the knowledge of sexual and reproductive rights and risky sexual practices in a sample of adolescents from the State of Campeche, in southeastern Mexico; for which an ad hoc 
questionnaire was applied in order to collect information on risky sexual behaviors and knowledge about sexual and reproductive rights to a sample of 490 high school students. Statistically significant relationships were found between knowledge of sexual and reproductive rights and substance use; as well as between the risk of unintended pregnancies, the risk of contracting STIs and receiving violence. No statistically significant differences were found in the comparison analysis between the sexes.

Keywords: Risky sexual practices, sexual rights, reproductive rights, adolescentes.

\section{INTRODUCCIÓN}

En el 2015 se llevó a cabo la Encuesta Intercensal en México que registró a más de 30.6 millones de jóvenes de entre 15 a 29 años, que representa al 25.7\% del total de la población (INEGI, 2017). Papalia (2012) define a la adolescencia como el periodo del desarrollo que va desde los 11 a 19 años y que comprende un conjunto de cambios en el individuo, cambios físicos, cognitivos, emocionales y sociales. En la adolescencia, la salud y el desarrollo físico, psicológico y social están estrechamente interrelacionados entre sí. Los cambios sexuales y corporales que se producen durante la adolescencia se acompañan de importantes cambios psicosociales, por lo que esta etapa es decisiva en el camino hacia la edad adulta.

De acuerdo con el Fondo de Población de las Naciones Unidas (UNFPA) las condiciones en las que adolescentes y jóvenes toman decisiones sobre su sexualidad, los elementos y servicios a los que tienen acceso, así como las oportunidades de educación y desarrollo disponibles, impactan significativamente tanto en la calidad de vida del individuo como en las tendencias poblacionales de las próximas décadas (UNFPA México, 2014). Es fundamental la presencia y el fácil acceso a los servicios de salud sexual y reproductiva evaluados como accesibles, equitativos y adecuados para que niñas, niños y adolescentes puedan ejercer sus derechos.

La Conferencia Internacional de Población y Desarrollo del Cairo (1994) reconoce el derecho de las y los adolescentes a la educación sexual y a servicios de salud adecuados para ellos. Los derechos humanos tienen el objetivo de otorgar protección a las personas de manera individual y grupal, contra faltas y actos que impidan o limiten sus libertades y derechos fundamentales (Galdos Silva, 2013). La ley respalda al individuo y se encarga que los Gobiernos e instituciones independientes hagan lo pertinente a ella y limita lo que pueda atentar la dignidad humana; los derechos humanos pertenecen a las garantías jurídicas universales y se caracterizan por ser igualitarios, individuales e independientes.

Se encontró información acerca de mitos relacionados con la cultura con respecto a la sexualidad, el hecho de no planear tener relaciones sexuales y terminar teniéndolas sin la precaución requerida a pesar de tener la información acerca de los riesgos implicados. 
El derecho a la salud sexual y reproductiva incluye otros derechos humanos como el derecho a la protección contra la violencia, el derecho de las mujeres a decidir en relación con su cuerpo sin coacción, también están presentes las garantías individuales al acceso a servicios, información e insumos de salud sexual y reproductiva, que sean de calidad y de precios accesibles (Cespedes \& Robles, 2016)

Los derechos sexuales y reproductivos son aquellos derechos que posee cada individuo a la toma de decisiones reproductivas, incluyendo la elección de casarse y de determinar el número y el espaciamiento de sus hijos, y el momento de tenerlos; la seguridad sexual y reproductiva, incluyendo el derecho de no padecer violencia ni coacción sexuales; estar informado y tener acceso a métodos de planificación familiar de su propia elección seguros, efectivos, asequibles y aceptables.

En América Latina y el Caribe, las mujeres jóvenes son más propensas a situaciones de salud sexual y reproductiva, como el inicio temprano de las relaciones sexuales, la maternidad temprana, infecciones de transmisión sexual, abortos en condiciones de alto riesgo, violencia de género, entre otras (OMS, 2009).

El Fondo de las Naciones Unidas para la Infancia (UNICEF) da a conocer que en 11 países de América Latina y el Caribe por cada nueve mujeres entre 15 a 24 años al menos una inicia su vida sexual antes de los 15 años. De acuerdo con los datos recopilados por la UNICEF (2015) las relaciones sexuales prematuras son más frecuentes entre adolescentes que viven en zonas rurales, en situación de pobreza o que cuentan con un bajo nivel educativo.

El embarazo adolescente se ha convertido en un problema poblacional que amplía las brechas sociales y de género, actualmente muchas mujeres de 15 a 24 años que viven con sus parejas no planean tener más hijos, o no desean tenerlos en su futuro inmediato Por ello prevenir su ocurrencia y erradicar el embarazo infantil son los objetivos estratégicos que el Gobierno de la República demanda acciones integrales, mecanismos de atención profesionales, de alta calidad, con total cobertura y perspectiva de género.

El derecho a la salud sexual y reproductiva se basa en los derechos humanos a la vida, la libertad y la seguridad personal, la igualdad de tratamiento, la privacidad, la educación y el desarrollo, la igualdad de género y el más alto nivel posible de salud física y mental.

Esta investigación tiene como propósito hacer énfasis en la importancia y acción de la ampliación a diversos sectores, tales como escuela e instituciones de salud, que tiene la función de ofrecer información básica de manera accesible e inmediata para una mayor promoción de métodos de protección y la reducción de prácticas sexuales de riesgo que generan problemáticas causales directas como lo son las altas tasas de infecciones de transmisión sexual y embarazo adolescente. 
Los datos obtenidos en la investigación estarán a disposición de la Universidad Autónoma de Campeche y al estado de Campeche para la concientización de los derechos sexuales y reproductivos con respecto a las prácticas sexuales de riesgo.

\section{MATERIALES Y MÉTODO}

\subsection{DISEÑO}

Se trató de una investigación con enfoque cuantitativo, de alcance descriptivo-correlacional al y diseño transversal.

\subsection{PARTICIPANTES}

La muestra fue no probabilística por conveniencia conformada por un total de 490 personas estudiantes de bachillerato en el Estado de Campeche en el sureste de México, la edad fue entre los 15 y 22 años ( $\square=18)$. La muestra se distribuyó en función de un $40.82 \%$ de hombres y un $59.18 \%$ de mujeres.

\subsection{INSTRUMENTO}

Se aplicó el cuestionario "Derechos Sexuales y Prácticas Sexuales de Riesgo" elaborado por los autores de la investigación, mismo que consta de dos apartados, el primero contiene preguntas enfocadas a los derechos sexuales y el segundo a las prácticas sexuales de riesgo.

Se obtuvo información relacionada a cinco aspectos:

1. Riesgo de ITS. En el cual se recopilo información sobre prácticas sexuales de riesgo que pueden derivar en Infecciones de Transmisión Sexual (ITS).

2. Riesgo de violencia. Mismo que hace referencia a conductas que suponen un riesgo a que la persona sea receptora de violencia.

3. Consumo de sustancias. Donde se recopiló información acerca de sustancias consumidas y su frecuencia.

4. Falta de información. Haciendo referencia a la falta de acceso a información confiable sobre sexualidad.

5. Derechos Sexuales y reproductivos. Donde se obtuvo información que alude al conocimiento de estos derechos.

\subsection{CONSIDERACIONES ÉTICAS}

Todas las personas que participaron lo hicieron por voluntad propia previo consentimiento informado. En el caso de las personas menores de edad, se solicitó un consentimiento informado también 
a quien tuviera la tutela. En ningún momento del proceso de la investigación se solicitó información que permitiera la identificación de quienes participaron.

El estudio se realizó de acuerdo con la Declaración de Helsinki, y el protocolo fue aprobado por el Comité de ética e investigación de la Facultad de Humanidades y del Departamento de Posgrado e Investigación de la Universidad Autónoma de Campeche. Todos los procedimientos seguidos estuvieron de acuerdo con los estándares éticos del comité responsable de experimentación humana de la Universidad Autónoma de Campeche, México; el código nacional de ética para la investigación psicológica, la ley de salud nacional y local y la Declaración de Helsinki de 1975, revisada en 2000.

\section{RESULTADOS}

Tabla 1 Comparación de medias con prueba t de factores por sexo de los participantes

\begin{tabular}{|c|c|c|c|c|c|}
\hline \multirow{2}{*}{ Factor } & \multicolumn{2}{|c|}{ Medias } & \multirow{2}{*}{$\begin{array}{l}\text { Diferencia } \\
\text { de medias }\end{array}$} & \multirow[b]{2}{*}{$\mathrm{t}$} & \multirow[b]{2}{*}{$\mathrm{p}$} \\
\hline & $\begin{array}{c}\text { Mujeres } \\
\mathrm{N}=290\end{array}$ & $\begin{array}{c}\text { Hombres } \\
\mathrm{N}=200\end{array}$ & & & \\
\hline Riesgo ITS & 14.65 & 15.10 & 0.45 & .485 & .630 \\
\hline Riesgo de violencia & 20.20 & 18.20 & 2.00 & -1.319 & .194 \\
\hline Consumo de sustancias & 25.23 & 25.50 & 0.27 & .151 & .881 \\
\hline Riesgo de embarazo & 57.37 & 55.50 & 1.87 & -1.264 & .213 \\
\hline $\begin{array}{l}\text { Falta de Información } \\
\text { Conocimiento sobre }\end{array}$ & 22.68 & 21.65 & 1.03 & -.896 & .375 \\
\hline $\begin{array}{l}\text { Derechos Sexuales y } \\
\text { Reproductivos }\end{array}$ & 9.13 & 8.30 & 0.83 & -.693 & .493 \\
\hline
\end{tabular}

Tras un análisis por sexo de las variables estudiadas se encontró que, los hombres presentaron medias más altas en comparación con las mujeres en el riesgo de contraer una infección de transmisión sexual y en el consumo de sustancias. Mientras que las mujeres obtuvieron medias más altas en el riesgo de recibir violencia, la práctica de conductas de riesgo para embarazos no planeados, la falta de información sexual confiable, no obstante, también obtuvieron una media más alta en relación con el conocimiento sobre derechos sexuales y reproductivos.

La diferencia de medias más alta se encontró en el riesgo a recibir violencia, en donde las mujeres obtuvieron una media dos puntos por encima de los hombres; no obstante, ninguna de las diferencias entre sexos resultó estadísticamente significativa. 
Tabla 2 Comparación de medias con ANOVA por factores según el número de personas con las que se ha tenido relaciones sexuales/eróticas

\begin{tabular}{|c|c|c|c|c|c|c|c|c|}
\hline & \multicolumn{6}{|c|}{ Medias } & \multirow{2}{*}{$\mathrm{F}$} & \multirow{2}{*}{$\mathrm{p}$} \\
\hline & $\begin{array}{c}0 \\
\text { personas }\end{array}$ & $\begin{array}{c}1 \text { a } 3 \\
\text { personas }\end{array}$ & $\begin{array}{c}4 \text { a } 6 \\
\text { personas }\end{array}$ & $\begin{array}{c}7 \text { a } 10 \\
\text { personas }\end{array}$ & $\begin{array}{c}11 \text { a } 15 \\
\text { personas }\end{array}$ & $\begin{array}{c}+15 \\
\text { personas }\end{array}$ & & \\
\hline Riesgo ITS & 10.78 & 16.25 & 16.20 & 17.66 & 18.00 & 18.00 & 17.17 & $.000 *$ \\
\hline Riesgo de violencia & 20.71 & 18.15 & 18.60 & 20.66 & 18.00 & 31.00 & 14.04 & .242 \\
\hline Consumo de sustancias & 23.35 & 24.90 & 22.30 & 28.66 & 51.00 & 54.00 & 10.25 & $.000 *$ \\
\hline Riesgo de embarazo & 60.21 & 54.40 & 56.20 & 51.66 & 61.00 & 65.00 & 4.39 & $.003 *$ \\
\hline $\begin{array}{l}\text { Falta de Información } \\
\text { Conocimiento sobre }\end{array}$ & 23.42 & 21.35 & 22.30 & 20.66 & 28.00 & 23.00 & .965 & .450 \\
\hline $\begin{array}{c}\text { Derechos Sexuales y } \\
\text { Reproductivos }\end{array}$ & 7.28 & 9.35 & 11.10 & 9.00 & .00 & 4.00 & 2.921 & $.023 *$ \\
\hline
\end{tabular}

*Dato estadísticamente significativo

$\mathrm{Al}$ analizar las variables en relación con el número de personas con las que se ha tenido relaciones sexuales/eróticas, se encontraron diferencias estadísticamente significativas en el riesgo a contraer ITS, el consumo de sustancias, el riesgo de embarazos no planeados y el conocimiento sobre derechos sexuales y reproductivos.

En el caso del riesgo a contraer ITS, consumo de sustancias y riesgo de embarazos no planeados, los grupos de personas que han mantenido relaciones sexuales/eróticas con más de 11 personas fueron quienes obtuvieron las medias más altas. En el caso del conocimiento sobre derechos sexuales y reproductivos, la media más alta fue obtenida por el grupo de 4 a 6 personas.

Tabla 3 Comparación de medias con ANOVA por factores según tipo de actividad sexual/erótica

\begin{tabular}{|c|c|c|c|c|c|c|}
\hline & \multicolumn{4}{|c|}{ Medias } & \multirow{2}{*}{$\mathrm{F}$} & \multirow{2}{*}{$\mathrm{p}$} \\
\hline & $\begin{array}{l}\text { Su mismo } \\
\text { sexo/género }\end{array}$ & $\begin{array}{c}\text { Del otro } \\
\text { sexo/género }\end{array}$ & Ambas & Ninguna & & \\
\hline Riesgo ITS & 15.80 & 14.92 & 17.22 & 10.71 & 8.11 & $.000 *$ \\
\hline Riesgo de violencia & 21.40 & 17.50 & 22.55 & 21.42 & 2.97 & $.042 *$ \\
\hline Consumo de sustancias & 26.20 & 24.67 & 27.77 & 23.85 & .427 & .735 \\
\hline Riesgo de embarazo & 54.00 & 56.35 & 56.66 & 59.42 & 1.451 & .230 \\
\hline Falta de Información & 23.80 & 22.14 & 22.33 & 21.57 & .314 & .815 \\
\hline $\begin{array}{l}\text { Conocimiento sobre Derechos } \\
\text { Sexuales y Reproductivos }\end{array}$ & 8.60 & 8.53 & 9.44 & 9.14 & .134 & .939 \\
\hline
\end{tabular}

*Dato estadísticamente significativo

En cuanto al análisis por actividad sexual/erótica, se encontraron diferencias estadísticamente significativas en el riesgo de contraer ITS y el riesgo de recibir violencia, en ambos casos, las personas que mantienen relaciones sexuales/eróticas con personas de ambos sexos son quienes presentaron las medias más altas. 
Tabla 4 Análisis correlacional entre las variables estudiadas

\begin{tabular}{|c|c|c|c|c|c|c|c|c|c|}
\hline & & 1 & 2 & 3 & 4 & 5 & 6 & 7 & 8 \\
\hline \multirow[t]{2}{*}{1 Edad } & $\mathrm{R}$ & 1 & -.091 & -.155 & -.116 & $\begin{array}{c}- \\
.235\end{array}$ & $-.336^{*}$ & $.300^{*}$ & \\
\hline & $\mathrm{P}$ & & .536 & .287 & .427 & .103 & .018 & .036 & .012 \\
\hline \multirow{2}{*}{2 Parejas sexuales } & $\mathrm{R}$ & & & .080 & $.491^{* *}$ & $\begin{array}{c}- \\
.025\end{array}$ & -.134 & $.636^{* *}$ & -.001 \\
\hline & $\mathrm{P}$ & & & .584 & .000 & .863 & .357 & .000 & .996 \\
\hline 3 Riesgo & $\mathrm{R}$ & & & & $.348^{*}$ & .018 & $.322^{*}$ & -.140 & -.159 \\
\hline Violencia & $\mathrm{P}$ & & & & .014 & .902 & .024 & .338 & .276 \\
\hline 4 Consumo & $\mathrm{R}$ & & & & & .103 & .214 & $.309^{*}$ & $-.449^{* * *}$ \\
\hline De Sustancias & $\mathrm{P}$ & & & & & .481 & .140 & .031 & .001 \\
\hline 5 Falta de & $\mathrm{R}$ & & & & & & $.284^{*}$ & -.061 & -.135 \\
\hline Información & $\mathrm{P}$ & & & & & & .048 & .675 & .355 \\
\hline 6 Riesgo de & $\mathrm{R}$ & & & & & & & $-.351^{*}$ & -.184 \\
\hline Embarazo & $\mathrm{P}$ & & & & & & & .013 & .207 \\
\hline 7 Riesgo & $\mathrm{R}$ & & & & & & & & .201 \\
\hline ITS & $\mathrm{P}$ & & & & & & & & .166 \\
\hline
\end{tabular}

8 Derechos sexuales y

reproductivos

*Dato estadísticamente significativo

Tras un análisis de correlación se determinó una relación estadísticamente significativa entre el conocimiento de los derechos sexuales y reproductivos y la edad, en donde se establece que aquellas personas de mayor edad tienen mayor conocimiento de estos derechos. Por otra parte, se encontraron relaciones significativas entre el número de parejas sexuales y el consumo de sustancias con el riesgo de contraer ITS, ambas relaciones positivas concluyendo que, a mayor número de parejas sexuales y mayor consumo de sustancia, mayor riesgo de contraer alguna ITS. La falta de información sexual confiable se relacionó de manera positiva con el riesgo de embarazos no planeados en tanto que, a mayor falta de información, mayor riesgo de embarazos no planeados.

\section{DISCUSIÓN}

Los resultados de esta investigación muestran relación entre la falta de información sexual y el riesgo de embarazos no planeados, la cual refiere que entre mayor sea la falta de información sexual posean las personas, habrá mayor riesgo de embarazos no planeados. Ospina \& Castaño (2009) mencionan que las limitaciones para acceder a los servicios, a los métodos de planificación familiar y la desinformación de temas asociados, son barreras para alcanzar los objetivos de la salud sexual y reproductiva, estas barreras están relacionadas a la falta de medios idóneos para que el individuo haga valer sus derechos. Por su parte Casas V. \& Cabezas (2016) exponen que los derechos sexuales y reproductivos se relacionan con el derecho que posee cada individuo a decidir libremente el número y espaciamiento entre los hijos e hijas, además de tener acceso a información y medios para ejercer la autonomía. 
En lo que respecta a Calafat, Juan, Becoña, Mantecón, \& Ramón (2009) encontraron que de acuerdo con su muestra el 62,3\% de los jóvenes piensan que estar bajo los efectos del alcohol y otras drogas les afecta a ellos en no tomar medidas contra las infecciones de transmisión sexual, el 76,6\% de la muestra a mantenido relaciones sexuales bajo los efectos del alcohol y el 55,2\% las ha mantenido bajo los efectos de alguna otra droga. Así mismo, se encontró que los jóvenes que se identifican como bisexuales tienen una diferencia no significativa del 36,4\% de contraer ITS. Contrario a esto, la presente investigación obtuvo como resultado una relación significativa en cuanto a las personas que mantienen relaciones sexuales/ eróticas con personas de ambos sexos corren más riesgo de contraer infecciones de transmisión sexual y de sufrir violencia.

En cuanto a los resultados de esta investigación sobre la relación entre el número de parejas sexuales, el consumo de alcohol y el riesgo de contraer ITS, se halló que entre más parejas sexuales tengas y mayor sea el consumo de sustancias, los riesgos de contraer alguna ITS aumenta. Resultados que coinciden con la investigación de Palacios \& Álvarez (2018) realizada en México respecto a la relación del número de parejas sexuales y el riesgo de adquirir alguna ITS, en dónde encontraron que en aquellas personas que mantienen más de 3 parejas sexuales, existe un mayor riesgo de contraer una infección de transmisión sexual.

Otro de los resultados obtenidos en la investigación fue la relación que existe del riesgo elevado que tienen de recibir violencia aquellas personas que mantienen relaciones con parejas de ambos sexos, sin embargo, no se encontró información similar al respecto.

En conclusión, no se encontraron diferencias significativas en esta investigación en relación con el sexo y la práctica de riesgo como: el consumo de sustancias, riesgo de ITS, embarazos no planeados, violencia y la falta de información. Contrario a esto, existe una relación significativa entre la edad y el conocimiento de los derechos sexuales, a mayor edad mayor será el conocimiento de los derechos sexuales y reproductivos; además, que la falta de información entre más presente esté, mayor será el riesgo de un embarazo no planeado. Por otra parte, el riesgo de adquirir alguna infección de transmisión sexual se relaciona significativamente con el número de parejas sexuales, el consumo de alcohol y el mantener relaciones sexuales/ eróticas con personas de ambos sexos, lo que significa que tener una mayor cantidad de parejas sexuales, que el consumo de alcohol aumente y mayor sea la cantidad de con personas de ambos sexos con las que se mantengan relaciones, se elevan las posibilidades de contraer una ITS.

Los resultados de la presente investigación aportan información relevante acerca del riesgo al que están expuestos las y los jóvenes adolescentes de adquirir alguna infección de trasmisión. Esta información ayuda a conocer cuáles son las prácticas sexuales de riesgo que están presentes en las y los 
jóvenes universitarios para poder implementar medidas de prevención que fomenten la salud sexual reproductiva responsable.

Dentro de las limitaciones que presenta esta investigación es necesario mencionar que la muestra se limita a estudiantes de la Universidad Autónoma de Campeche, se sugiere ampliar la muestra a otras universidades, instituciones no educativas como en el sector salud e incluir una población de adultez media y adultez tardía para realizar comparaciones en las diferentes etapas del desarrollo.

\section{AGRADECIMIENTOS}

Agradecemos a las autoridades de las escuelas preparatorias de la Universidad Autónoma de Campeche por su colaboración en este trabajo, también a la Facultad de Humanidades y a los miembros del cuerpo académico "Desarrollo Humano" por su apoyo en la realización de este trabajo.

Los Autores declaramos no tener ningún conflicto de interés en la publicación del presente artículo. 


\section{REFERENCIAS}

Calafat, A., Juan, M., Becoña, E., Mantecón, A., \& Ramón, A. (2009). Sexualidad de riego y consumo de drogas en el contexto recreativo. Una perspectiva de género. Psicothema, 21(2), 227-233.

Casas V., M., \& Cabañas, G. (2016). Working papers CDH. Obtenido de http://repositorio.uchile.cl/bitstream/handle/2250/142698/Los-derechos-sexuales-yreproductivos.pdf? sequence $=1$ \&isAllowed $=\mathrm{y}$

Céspedes, C., \& Robles, C. (2016). Niñas y adolescentes en América Latina y el Caribe: Deudas de igualdad.Naciones Unidas.

Escamilla, M., Guzmán, R. (2017). Educación sexual en México ¿misión de la casa o de la escuela?. Educación y Salud Boletín Científico de Ciencias de la Salud del ICSA. Vol. 5 (10). Recuperado de https://www.uaeh.edu.mx/scige/boletin/icsa/n10/e1.htm

Galdos,S. (2013). La conferencia de El Cairo y la afirmación de los derechos sexuales y reproductivos, como base para la salud sexual y reproductiva. Revista peruana de medicina experimental y salud pública, volumen (30), pp. $\quad 455 . \quad$ Recuperado de https://rpmesp.ins.gob.pe/index.php/rpmesp/article/view/283/2185

Instituto Nacional de Estadística y Geografía. (2017). Estadísticas a propósito del... Día Internacional de la Juventud (12 de Agosto). Recuperado de: http://consulta.mx/index.php/estudios-einvestigaciones/otros-estudios/item/702-inegi-estadisticas-a-proposito-del-dia-internacional-de-lajuventud-12-de-agosto

OMS (Organización Mundial de la Salud) (2009), Promoting adolescent sexual and reproductive health through schools in low income countries: an information brief, Department of Child and Adolescent Health and Development, Ginebra.

Ospina M., D. E., \& Castaño L., R. A. (2009). Producción científica sobre derechos sexuales y reproductivos, Colombia 1994-2004. Investigación y Educación en Enfermería, XXVII(2), 181-204.

Palacios, J., \& Álvarez, M. (2018). Consumo de drogas asociadas al contagio de infecciones de transmisión sexual en jóvenes de México. Health and Addictions, 18(2), 111-120.

Papalia, D. E., Feldman, R. D., \& Martorell, G. (2012). Desarrollo Humano. México, D. F.: McGrawHill.

UNFPA México. (2012). UNFPA México. Obtenido de Salud Sexual y Reproductiva en adolescentes y jóvenes: https://mexico.unfpa.org/es/topics/salud-sexual-y-reproductiva-en-adolescentes-y-jóvenes

UNICEF. (2015). UNICEF. Obtenido de Una aproximación a la situación de adolescentes y jovenes en América Latina y el Caribe a partir de evidencia cuantitativa reciente: https://www.unicef.org/lac/sites/unicef.org.lac/files/2018-

04/UNICEF_Situacion_de_Adolescentes_y_Jovenes_en_LAC_junio2105.pdf 